\title{
Construction and analysis of a IncRNA (PWRN2)-mediated ceRNA network reveal its potential roles in oocyte nuclear maturation of patients with PCOS
}

Xin Huang ${ }^{*+} \mathbb{D}$, Jiaping Pan $^{\dagger}$, Bi Wu and Xiaoming Teng ${ }^{*}$

\begin{abstract}
Background: Polycystic ovary syndrome (PCOS) is a common endocrine and metabolic disorder in women. An IncRNA, namely, Prader-Willi region nonprotein coding RNA 2 (PWRN2), was up-regulated in the cumulus cells of patients with PCOS. However, the molecular mechanism of PWRN2 in PCOS remains largely unknown.

Methods: In this study, the expression levels of PWRN2 were tested in cumulus cells through qRT-PCR analysis to confirm its potential roles in oocyte nuclear maturation of PCOS. A PWRN2-mediated ceRNA network was constructed based on three microarray datasets to investigate the molecular mechanism of PWRN2 in oocyte development of patients with PCOS. The direct interactions of the candidate genes of the ceRNA network were also demonstrated by dual-luciferase reporter assay.

Results: PWRN2 was found to be associated with oocyte nuclear maturation in patients with PCOS in contrast to that in normal patients. Based on the microarray data, 176 IncRNAs (118 up-regulated and 58 down-regulated) and 131 mRNAs (84 up-regulated and 47 down-regulated) were identified to be regulated by PWRN2. A PWRN2-miR92b-3p-TMEM120B ceRNA network was constructed based on results of analysis of the combined three microarray datasets (IncRNA+mRNA microarray in KGN/shPWRN2 in this study, miRNAs microarray and IncRNA+mRNA microarray in PCOS cumulus cells reported in previous studies). The coexpression characteristics of the genes (PWRN2, miR-92b-3p and TMEM120B) were detected in the cumulus cells of cumulus-oocyte complexes at different nuclear maturity stages in PCOS. These results are in accordance with the ceRNA hypothesis. Moreover, luciferase activity assay revealed that miR-92b-3p directly binds to PWRN2 and targets TMEM120B.

Conclusions: PWNR2 plays important roles in oocyte nuclear maturation in PCOS by functioning as a ceRNA to reduce the availability of miR-92b-3p for TMEM120B target binding during oocyte maturation in PCOS. Our findings would provide new information and clarify abnormal oocyte development in PCOS.
\end{abstract}

Keywords: ceRNA, Oocyte maturation, Polycystic ovary syndrome, PWRN2, miR-92b-3p, TMEM120B

\footnotetext{
* Correspondence: huangxin92129@163.com; tengxiaoming@hotmail.com

${ }^{+}$Xin Huang and Jiaping Pan contributed equally to this work.

Department of Assisted Reproductive Medicine, Shanghai First Maternity and Infant Hospital, Tongji University School of Medicine, 2699 Gaoke Road West, Shanghai 200001, People's Republic of China
}

(c) The Author(s). 2018 Open Access This article is distributed under the terms of the Creative Commons Attribution 4.0 International License (http://creativecommons.org/licenses/by/4.0/), which permits unrestricted use, distribution, and reproduction in any medium, provided you give appropriate credit to the original author(s) and the source, provide a link to the Creative Commons license, and indicate if changes were made. The Creative Commons Public Domain Dedication waiver (http://creativecommons.org/publicdomain/zero/1.0/) applies to the data made available in this article, unless otherwise stated. 


\section{Background}

Polycystic ovary syndrome (PCOS) is a common endocrinopathy in women of reproductive age [1] and accounts for approximately $75 \%$ of anovulatory infertility disorders [2]. The phenotype of PCOS is variable and includes hyperandrogenism, menstrual irregularity and polycystic ovarian morphology [3]. Patients suffering from PCOS are often diagnosed with obesity, hirsutism, insulin resistance, increased risk of endometrial cancer, metabolic syndrome [4], type 2 diabetes (T2D) and cardiovascular diseases $[5,6]$. Although the aetiology of PCOS remains unclear, most researchers regard PCOS as multifactorial and suggest that genetic factors play a pivotal role in its development and maintenance $[7,8]$. Many studies have reported gene expression profiles based on tissues (i.e. theca cells [9], ovaries [10, 11], oocytes [12] and cumulus cells [13]) from controls and patients with PCOS. Genes associated with PCOS are involved in the insulin receptor signalling pathway, steroid biosynthesis and regulation of gonadotropin secretion [14]. However, the mechanism by which these genes are regulated has not been thoroughly elucidated.

Long noncoding RNAs (lncRNAs) are defined as noncoding RNAs with greater than 200 base pairs [15]. LncRNAs were previously regarded as transcriptional 'noise' without biological functions [16]; however, increasing lines of evidence indicate that lncRNAs play key roles in normal development and diseases [17]. To date, several reports have demonstrated that lncRNAs may function in PCOS-related diseases, including T2D, obesity and cardiac diseases. For instance, a $\beta$-cell-specific IncRNA (HI-LNC25) is dysregulated in T2D by down-regulating the mRNA expression levels of GLIS3 (Kruppel-like zinc finger transcription factor) [18].

In our previous research [19], we used microarrays [Agilent human lncRNA+mRNA Array v2.0 $(4 \times$ $180 \mathrm{~K}$ format)] to describe lncRNA profiles in cumulus cells isolated from 10 patients (five patients with PCOS and five normal women). A total of 623 lncRNAs were differentially expressed in PCOS and may contribute to its occurrence [19].Among these lncRNAs, Prader-Willi region nonprotein coding RNA 2 (PWRN2) (transcript ID: ENST00000567246.1), which is expressed in the testes and is up-regulated after meiosis during spermatogenesis [20], was found to be up-regulated (3.11-fold) in the cumulus cells of patients with PCOS. Oocyte nuclear maturation has two meiosis resumption processes at the MI (the first meiosis resumption) and MII (the second meiosis resumption) stages. Hence, PWRN2 may be associated with oocyte nuclear maturation in PCOS. In addition, abnormal folliculogenesis is regarded as a common characteristic of PCOS although its clinical and biochemical signs are typically heterogeneous [21, 22].
Thus, studying the abnormal regulatory mechanisms in oocyte development of PCOS is important.

Increasing lines of evidence suggest that lncRNAs function as miRNA sponges or competing endogenous RNAs (ceRNAs) to reduce the availability of miRNAs for mRNA target binding $[23,24]$. In the present study, we confirmed the potential roles of PWRN2 in oocyte nuclear maturation of PCOS. We then constructed a PWRN2- mediated ceRNA network by analysing three microarray datasets (lncRNA+mRNA microarray in PCOS cumulus cells [19], miRNAs microarray in PCOS cumulus cells [25] and IncRNA+mRNA microarray in KGN/shPWRN2 in this study) to investigate the mechanism of PWRN2. Results revealed the potential roles of ceRNA in oocyte maturation in PCOS. This work highlights a novel mechanism of oocyte nuclear maturation in PCOS and provides new targets for PCOS treatment.

\section{Methods}

\section{Patients and IVF treatment}

The inclusion criteria for the recruited patients (PCOS and normal) and the methods for collecting CCs were based on our previous reports [19, 26]. This study was approved by the Institutional Ethical Review Board of Tongji University School of Medicine. Sixty participants (30 patients with PCOS and 30 normal) who were referred to our centre for IVF were included in this study after obtaining written informed consent. All patients had no history of taking drugs that affect glucose and lipid metabolism and did not have any known medical conditions or diseases, such as Cushing's syndrome, congenital adrenal hyperplasia, androgen-secreting tumours and endometriosis. Patients with PCOS were diagnosed according to the revised Rotterdam European Society of Human Reproduction and Embryology/ American Society for Reproductive Medicine Criteria [3]. The patients were required to present at least two of the following criteria: chronic oligo-ovulation or anovulation, androgen excess and polycystic ovaries. The inclusion criteria for the recruited patients in this study were as follows: age $<36$ years, BMI ranging between 20 and $26 \mathrm{~kg} / \mathrm{m}^{2}$, basal serum LH/FSH more than 2.0 , serum testosterone more than $0.5 \mathrm{ng} / \mathrm{mL}$, antral follicle count ranging between 18 and 35 and number of obtained oocytes ranging between 12 and 28 per cycle. Control patients had regular menstrual cycles, normal ovary sonographs and normal ovulation with bilateral tube occlusion, were nondiabetic and showed no clinical signs of hyperandrogenism and anovulation. The clinical characteristics of control patients and those with PCOS are summarised in Table 1.

Patients in both groups received an agonist protocol as described previously [27]. All patients received the GnRH agonist triptorelin acetate $(0.05 \mathrm{mg} /$ day, Diphereline; 
Table 1 Clinical characteristics of patients

\begin{tabular}{llll}
\hline & Normal $(\mathrm{n}=30)$ & PCOS $(\mathrm{n}=30)$ & $P$-value \\
\hline Age $($ years $)$ & $33.6 \pm 2.2$ & $32.6 \pm 3.1$ & $\mathrm{NS}$ \\
BMI $\left(\mathrm{kg} / \mathrm{m}^{2}\right)$ & $21.4 \pm 1.6$ & $21.6 \pm 1.5$ & $\mathrm{NS}$ \\
$\mathrm{FSH}(\mathrm{mlU} / \mathrm{ml})$ & $6.51 \pm 1.2$ & $5.41 \pm 1.3$ & $<0.05$ \\
$\mathrm{LH}(\mathrm{mlU} / \mathrm{ml})$ & $4.30 \pm 1.4$ & $11.91 \pm 2.6$ & $<0.001$ \\
Basal LH/FSH & $0.65 \pm 0.2$ & $2.52 \pm 0.61$ & $<0.001$ \\
E2 $(\mathrm{pg} / \mathrm{ml})$ & $38.5 \pm 4.3$ & $41.3 \pm 9.5$ & $\mathrm{NS}$ \\
Testosterone $(\mathrm{ng} / \mathrm{ml})$ & $0.12 \pm 0.05$ & $0.68 \pm 0.05$ & $<0.001$ \\
Progesterone $(\mathrm{ng} / \mathrm{ml})$ & $0.55 \pm 0.22$ & $0.70 \pm 0.23$ & $\mathrm{NS}$ \\
Antral follicle count & $10.2 \pm 1.1$ & $24.1 \pm 3.7$ & $<0.001$ \\
Oocytes obtained & $8.5 \pm 3.0$ & $17.8 \pm 5.2$ & $<0.001$ \\
No of Mll oocytes & $6.3 \pm 1.8$ & $14.5 \pm 4.6$ & $<0.001$ \\
\hline
\end{tabular}

Data are the mean \pm SEM, NS not statistically significant

$B M I$ body mass index, E2 oestradiol, FSH follicle-stimulating hormone,

LH luteotropic hormone, $P C O S$ polycystic ovary syndrome

Ipsen Pharma Biotech, Paris, France) subcutaneously starting at the mid-luteal phase. Once adequate pituitary down-regulation was confirmed [serum LH levels < $3.0 \mathrm{ng} / \mathrm{mL}$ and serum estradiol $\left(\mathrm{E}_{2}\right)$ levels $<30 \mathrm{pg} / \mathrm{mL}$, the patients received recombinant FSH (150-187.5 IU; Gonal-f, Follitropin Alfa, Serono) subcutaneously for COS. When two or more follicles were at least $18 \mathrm{~mm}$ in diameter and the serum $\mathrm{E}_{2}$ levels were at least $300 \mathrm{pg} / \mathrm{mL}$ per dominant follicle, all patients received $250 \mu \mathrm{g}$ of hCG (Profasi, Serono).

\section{Retrieval of cumulus cells}

Collection of CCs and assessment of oocytes were conducted as previously described [27, 28]. Cumulus-oocyte complex (COC) retrieval was performed by vaginal puncture under ultrasound echo-guidance $36 \mathrm{~h}$ after hCG administration. After COC retrieval, a portion of CCs surrounding a single oocyte was removed using a sharp needle. For RNA extraction, the cumulus cells were lysed in $80 \mu \mathrm{L}$ of lysis buffer (mirVana miRNA Isolation Kit; Ambion, Austin, TX, USA) and stored at $-80{ }^{\circ} \mathrm{C}$. For vector transfection and luciferase activity assay, the cumulus cells were firstly digested with trypsin and then cultured directly. Oocytes were further inseminated by ICSI and cultured in sequential media of SAGE (CooperSurgical, Leisegang Medical, Berlin) individually in $20 \mu \mathrm{L}$ of droplets covered with mineral oil. The embryos were transferred or vitrified on day 3 , and the other embryos were cultured to blastula stage on days 5-6.

\section{Assessment of oocyte and division of the groups of cumulus cells}

The morphological characteristics of the oocytes were individually recorded. The oocytes were denudated to assess the maturation stage before ICSI. Few of germinal vesicle (GV)-stage COCs (12 in patients with PCOS and only 3 in normal patients) were retrieved. We classified the COCs into two categories based on nuclear status: (i) MI/GV group: immature MI oocytes exhibiting no polar bodies (PB) or immature oocytes at the GV stage, and (ii) MII group: mature MII oocytes that extruded a clearly visible $\mathrm{PB}$. The corresponding cumulus cells were divided into $\mathrm{CC}_{\mathrm{MI} / \mathrm{GV}}$ and $\mathrm{CC}_{\mathrm{MII}}$ groups. Each group had $\geq$ three replicates. Each subgroup, containing at least 15 cumulus cells, represented a biological replicate. Each $\mathrm{CC}_{\mathrm{MI} / \mathrm{GV}}$ subgroup has one $\mathrm{CC}_{\mathrm{GV}}$.

\section{RNA extraction}

Total RNA was isolated using a Qiagen RNeasy Mini Kit (Qiagen, Hilder, Germany) according to the manufacturer's instructions. This RNA isolation kit significantly reduced contamination from genomic DNA and proteins. The purity and concentration of RNA were determined from OD260/280 readings using a spectrophotometer (NanoDrop ND-1000). RNA integrity was determined using $1 \%$ formaldehyde denaturing gel electrophoresis.

\section{qRT-PCR}

The expression levels of PWRN2 in the $\mathrm{CC}_{\mathrm{MI} / \mathrm{GV}}$ and $\mathrm{CC}_{\mathrm{MII}}$ groups of normal patients and those with PCOS were tested by qRT-PCR analysis to evaluate the correlation of changes in PWRN2 with oocyte maturation. The potential ceRNA network was constructed to investigate the action mechanism of PWRN2.The co-expression characteristics of the candidate genes of the ceRNA network were also tested in the CCs corresponding to oocytes at different nuclear maturity stages (MI/GV and MII) of patients with PCOS through qRT-PCR analysis.

Total RNA was reverse transcribed into cDNA by using a miScript Reverse Transcription Kit (Qiagen). qRT-PCR analysis was performed using SYBR green assay (Takara Bio, Inc., Dalian, China) according to the manufacturer's protocols. PCR was performed in a total reaction volume of $20 \mu \mathrm{L}$ containing $10 \mu \mathrm{L}$ of $2 \times$ QuantiTest SYBR Green PCR Master Mix, $1 \mu \mathrm{L}$ of cDNA template, $1 \mu \mathrm{L}$ of each primer and RNase-free water. The primers used in this study are listed in Additional file 1: Table S1. All reactions were performed using the ABI PRISM 7300 system. The amplification conditions were as follows: $10 \mathrm{~min}$ at $98^{\circ} \mathrm{C}$; 40 cycles of $15 \mathrm{~s}$ at $95{ }^{\circ} \mathrm{C}, 1 \mathrm{~min}$ at $60{ }^{\circ} \mathrm{C}$; and a final extension for 5 min at $72{ }^{\circ} \mathrm{C}$. Amplification efficiency was evaluated by standard curve analysis. PWRN2 and mRNA expression data were normalised to those of GAPDH. The miRNA expression data were normalised to U6. Each set of qRT-PCR reactions was repeated at least three times, and fold change in the expression of each gene was analysed by the $\Delta \Delta \mathrm{Ct}$ method [29]. 


\section{KGN cell culture}

PWRN2-regulated genes were identified using RNA interference technology to inhibit the expression of PWRN2 in KGN cell lines and eliminate differences in the genetic backgrounds of different patients with PCOS. KGN (RCB1154; RIKEN, Wako, Japan) is a steroidogenic human ovarian granulosa tumour cell line. KGN cells were cultured in 1:1 Dulbecco's modified Eagle's medium and Ham's F-12 medium (DMEM/ F12; Nissui Pharmaceutical, Tokyo, Japan) supplemented with $10 \%$ foetal bovine serum (FBS) and antibiotics (100 IU/mL penicillin and $100 \mu \mathrm{g} / \mathrm{mL}$ streptomycin). On the day before lentivirus transfection, KGN cells were placed into the medium without serum and incubated overnight.

\section{Construction of lentivirus shRNA and cell transfections}

Lentivirus shRNA construction and cell transfection were conducted using previously described methods $[30,31]$. We selected three target sequences to construct lentiviral shRNAs (LV-PWRN2-homo-502, LV-PWRN2-homo-1574 and LV-PWRN2-homo-1261) and included a negative control (LV-NC) (Table 2). The target sequences were used to design two complementary oligonucleotides, which were synthesised and cloned into pGLV3/H1/GFP + Puro Vector (GenePharma, China). The positive purified lentiviral shRNA-expressing plasmids were transfected with packaging plasmids into $293 \mathrm{~T}$ cells for lentivirus generation (GenePharma, China). The vectors described above were used to infect KGN cells. Stable KGN cell lines were selected using $3 \mu \mathrm{g} / \mathrm{mL}$ bulk puromycin-resistance culture (puromycin, Sigma, St Louis, MO, USA) for 5 days. Afterwards, the cells were examined microscopically for lentiviral GFP expression. The expression levels of PWRN2 in KGN/ shPWRN2 cells and the corresponding negative-control KGN cells were tested by qRT-PCR to validate the effects of RNA interference.

\section{Microarray hybridisation and data analysis}

For microarray analysis, three KGN/shPWRN2 cell lines with down-regulated $(\mathrm{FC}<0.5) \quad$ PWRN2 mRNA levels were selected as shPWRN2 groups. The corresponding three KGN cell lines with negative control vectors were

Table 2 Target sequences of lentiviral shRNAs for interfering PWRN2

\begin{tabular}{lll}
\hline LV-shPWRN2 & Site & Target sequences \\
\hline PWRN2-homo-502 & $502-522$ & 5'-GCCATTCGGTTACCATCTACT-3' \\
PWRN2-homo-1574 & $1574-1594$ & 5'-GCAAAGGAATTACCGTTAACA-3' \\
PWRN2-homo-1261 & $1261-1281$ & 5'-GGCAGAAAGCAATGAAGAAGA-3' \\
NC & Nonsense & 5'-TTCTCCGAACGTGTCACGT-3' \\
\hline
\end{tabular}

used as the control groups. The methods for RNA labelling, array hybridisation and data analysis were described in our previous report [19]. The purified RNA extracted from KGN/shPWRN2 samples or normal KGN cells was amplified and transcribed into fluorescent cDNA for hybridisation to the Agilent human lncRNA+ mRNA Array v4.0 $(4 \times 180 \mathrm{~K}$ format $)$ with each array containing probes interrogating approximately 41,000 human lncRNAs and approximately 34,000 human mRNAs. The lncRNA+mRNA array data were analysed to summarise, normalise and assess the quality of the data using GeneSpring software V11.5 (Agilent). To select differentially expressed genes, we used threshold values of $\geq 2.0$ - and $\leq-2.0$-fold changes and a BenjaminiHochberg corrected $P$ value of 0.05 . The data were $\log 2$ transformed and median centred by genes using the Adjust Data function of the CLUSTER 3.0 software. The data were further analysed using hierarchical clustering with average linkages. Finally, we visualised the tree using Java TreeView (Stanford University School of Medicine, Stanford, CA, USA). Microarray hybridisation and data analysis were performed by CapitalBio Corporation, Beijing, P. R. China.

\section{Gene ontology (GO) and KEGG pathway analyses}

$\mathrm{GO}$ analysis was performed to describe genes and gene product attributes in any organism (http://www.geneontology.org). This ontology covers three domains: biological processes, cellular components and molecular functions. The $P$ value denotes the significance of the GO term enrichment among differentially expressed genes ( $P$ value $\leq 0.05$ is recommended). For pathway analysis, we used the free web-based Molecular Annotation System 3.0 (MAS 3.0; http://bioinfo.capitalbio.com/ mas3/), which integrates three different open-source pathway resources: KEGG, BioCarta and GenMAPP. The significantly altered pathways were selected using the threshold of the $\mathrm{P}$ value and FDR (corrected $\mathrm{P}$ value) $<0.05$ derived from the hypergenomic test. GO and KEGG pathway analyses were performed by CapitalBio Corporation, Beijing, P. R. China.

\section{Construction of the PWRN2-mediated ceRNA network based on microarray data}

A potential PWRN2-mediated ceRNA network was constructed based on three microarray datasets to explain whether PWRN2 functions as miRNA sponges or ceRNAs. The putative miRNAs and mRNAs included in the construction of the PWRN2-mediated ceRNA network are as follows: (i) miRNA selection: miRNAs were predicted to possess PWRN2 binding sites by using miRanda v3.3a software and were compared with previous miRNA microarray data of PCOS; putative miRNAs with MREs in PWRN2 and identified from previous 
microarray data of PCOS were selected; and (ii) mRNA selection: the target genes of the miRNAs were predicted using miRbase (http://www.mirbase.org/) and compared with the lncRNA+mRNA microarray data from KGN cells with PWRN interference. The selected mRNAs met two criteria: 1) differentially expressed mRNAs identified from our IncRNA+mRNA microarray data; and 2) putative target genes of miRNAs with MREs in PWRN2.

\section{Luciferase reporter constructs and luciferase activity assay}

The direct interaction between the candidate genes of PWRN2-mediated ceRNA network was evaluated by luciferase activity assay. A luciferase reporter vector (pmirGLO Dual-Luciferase miRNA Target Expression Vector; Promega) was used for luciferase constructs. PWRN2 and the 3'UTR of TMEM120B were cloned by RT-PCR. PWRN2-WT, PWRN2-mutant and TMEM120B-3'UTR (WT and mutant) were constructed as previously reported [26, 32]. Cumulus cells of patients with PCOS were digested with $0.25 \%$ trypsin for $5 \mathrm{~min}$. The digestion was inhibited by adding $2.5 \mathrm{~mL}$ of DMEM: F12 containing $10 \%$ FBS and by incubating at room temperature for $5-10 \mathrm{~min}$. The cumulus cells were pooled in a sterile $15 \mathrm{~mL}$ conical tube on ice, centrifuged at $200 \mathrm{~g}$ for $10 \mathrm{~min}$, washed once with sterile saline, centrifuged again and resuspended in $3 \mathrm{~mL}$ of culture media. The number of viable cells was determined by trypan blue exclusion and ranged between 35 and 45\%. The cumulus cells were plated onto 24-well plates and allowed to grow for $\sim 24 \mathrm{~h}$ before transfection. The constructed reporter vectors (300 ng) were transfected into cells together with the miRNA and control mimics (100 nM) in Lipofectamine $2000(2 \mu \mathrm{L})$. The cells were lysed after $24 \mathrm{~h}$ of transfection. Luciferase activity was assayed using the Dual-Luciferase Reporter Assay System (Promega). Firefly luciferase activities were normalised to Renilla luciferase activities. The experiments were performed independently in triplicate.

\section{Statistical analysis}

All statistical analyses were performed using SPSS 17.0 software (SPSS Inc.) unless otherwise noted. Differences in the expression levels of the candidate genes of the PWRN2-mediated ceRNA network in cumulus cell samples were evaluated by two-tailed t-test. Differences were considered statistically significant at $P<0.05$.

\section{Results}

Expression levels of PWRN2 in cumulus cells according to oocyte nuclear maturity in normal patients and those with PCOS

The transcript levels of PWRN2 were detected in the $\mathrm{CC}_{\mathrm{MI} / \mathrm{GV}}$ and $\mathrm{CC}_{\mathrm{MII}}$ groups to evaluate the involvement of PWRN2 in the oocyte nuclear maturity in patients with PCOS $(n=30)$ and normal patients $(n=30)$. For PCOS, the mean transcript levels of PWRN2 in the $\mathrm{CC}_{\mathrm{MII}}$ group was significantly down-regulated by $4.55-$ fold compared with that in the $\mathrm{CC}_{\mathrm{MI} / \mathrm{GV}}$ group (1.006 \pm 0.028 versus $4.602 \pm 0.128$; Fig. $1 \mathrm{~A})$. In normal patients, the expression levels of PWRN2 exhibited no significant differences between the $\mathrm{CC}_{\mathrm{MII}}$ and $\mathrm{CC}_{\mathrm{MI} / \mathrm{GV}}$ groups $(1.02 \pm 0.029$ versus $0.98 \pm 0.049$; Fig. 1B). These results indicated that the PWRN2 expression pattern during oocyte maturation differed between normal patients and those with PCOS.

\section{Expression profiles of IncRNAs and mRNAs in KGN/ shPWRN2 cell lines}

To avoid the different genetic backgrounds of paitents with PCOS, we used KGN cell lines to identify PWRN2-regulated genes by RNA interference. Lentiviral shRNAs were constructed to inhibit PWRN2 expression in KGN cells. The results of qRT-PCR analysis indicated that the relative expression levels of PWRN2 mRNA were $0.23,0.25$ and 0.19 in KGN cells treated with LV-PWRN2-homo-502, LV-PWRN2-homo-1574 and LV-PWRN2-homo-1261, respectively, compared with those in the LV-NC group (Fig. 2A). These results indicated that the three lentiviral shRNAs effectively inhibited PWRN2 replication in KGN cells.

The lncRNA and mRNA expression profiles of KGN/ shPWRN2 cells infected with LV-PWRN2-homo-502, LV-PWRN2-homo-1574 or LV-PWRN2-homo-1261 were examined by microarrays and compared with those of the three KGN cell lines infected with control LV-NC. The raw microarray data were deposited in NCBI's Gene Expression Omnibus (GEO; http://www.ncbi.nlm.nih.gov/geo/) and can be accessed through the GEO series accession number GSE97772. The microarray analysis revealed thousands of lncRNAs and mRNAs expressed in $\mathrm{KGN} / \mathrm{sh} P W R N 2$.

The expression of 176 lncRNAs significantly changed in KGN/shPWRN2 cells relative to the control KGN cell lines. A total of 118 lncRNAs were up-regulated, whereas 58 lncRNAs were down-regulated. A total of 131 mRNAs significantly changed in the KGN/ shPWRN2 cell lines. About 84 mRNAs were up-regulated and 47 mRNAs were down-regulated (fold change $\geq 2.0, P<0.05)$. Clustering analysis of the microarrays based on the differentially expressed lncRNAs or mRNAs clustered the KGN/shPWRN2 groups and the control groups (Fig. 2B).

The top 20 up and down-regulated lncRNAs and mRNAs are listed in Tables 3 and 4, respectively. From the microarray data, the expression level of PWRN2 was down-regulated $(\mathrm{FC}=-2.526)$ in the $\mathrm{KGN} / \mathrm{sh} P W R N 2$ groups. The results were in concordance with the 


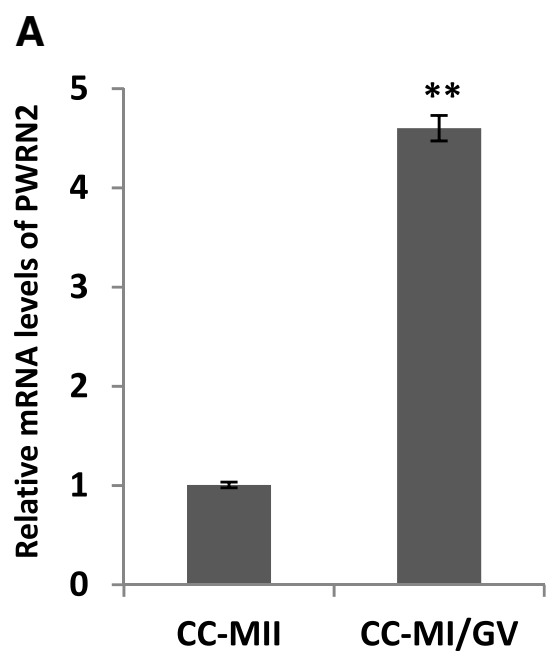

PCOS
B

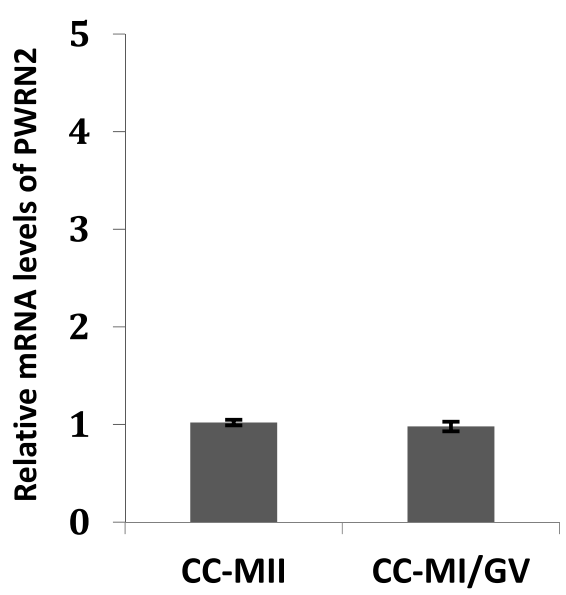

\section{Normal}

Fig. 1 Transcript levels of PWRN2 according to oocyte nuclear maturity in PCOS $(n=30)$ and normal patients $(n=30)$. Expression levels of PWRN2 in cumulus cells of different oocyte nuclear maturity stages (MI/GV stage and MII stage) of patients with PCOS (a) and normal patients (b). The signal intensity for PWRN2 is shown on the y-axis in arbitrary units determined by qRT-PCR analysis with GAPDH as an endogenous reference. * indicates a significant difference in gene expression between CC categories ( ${ }^{* *} P<0.01,{ }^{*} P<0.05$ ). The results are presented as means \pm SEM. $\mathrm{CC}_{\text {MI/GV: }}$ cumulus cells from oocytes at the MI or GV stage; $\mathrm{CC}_{\text {MII: }}$ cumulus cells from oocytes at the MII stage
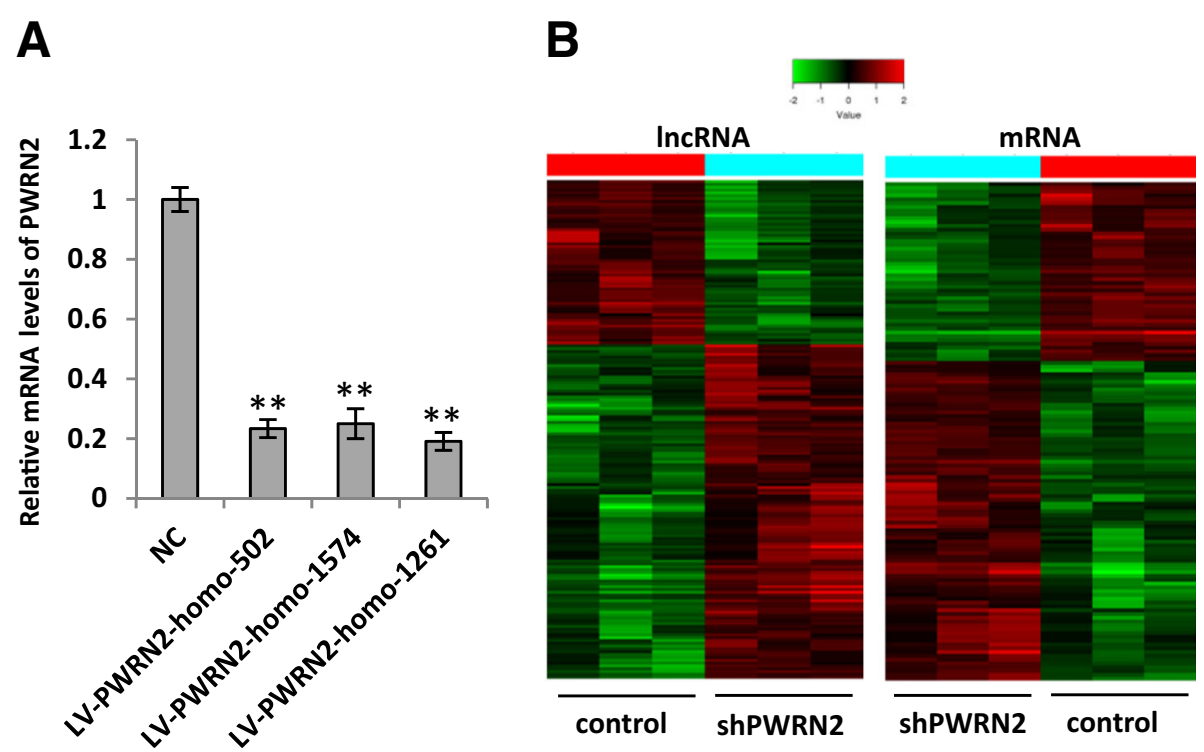

Fig. 2 Expression profiles of IncRNAs and mRNAs in KGN/shPWRN2 cell lines. a Relative expression of PWRN2 mRNA was examined in KGN cells infected with different lentiviral shRNAs (LV-PWRN2-homo-502, LV-PWRN2-homo-1574, LV-PWRN2-homo-1261, or LV negative control) using qRT$P C R$ analysis with GAPDH as an endogenous reference. The KGN cells treated with different lentiviral shRNAs are shown on the $x$-axis, and the relative change of $P$ WRN2/GAPDH is shown on the $y$-axis. Each set of qRT-PCR reactions was repeated at least three times. The results are presented as mean \pm SEM. ${ }^{* *}$ indicates $P<0.01$. $\mathbf{b}$ Cluster of InCRNAs and mRNAs that were dyexpressed in KGN/shPWRN2 cells. From this IncRNA + mRNA microarray data, 176 IncRNAs changed significantly in KGN/shPWRN2 cells compared with those in the control cell lines. Among these IncRNAs, 118 were up-regulated, whereas 58 IncRNAs were down-regulated; and 131 mRNAs changed significantly in the KGN/shPWRN2 cell lines. Among these, 84 mRNAs were up-regulated, and 47 mRNAs were down-regulated. The supervised hierarchical clustering of genes overexpressed in KGN/shPWRN2 cells is shown. Distinct signatures were observed in the KGN/shPWRN2 cells. The value of each gene was adjusted with a median-centring algorithm on a log scale; the colours indicate the relative gene expression in the red-green heat map. Pure black indicates 0.00 and represents no change in the median gene expression levels in all samples. Pure green indicates -2.00 and represents lower expression. Pure red indicates +2.00 and relatively higher expression 
Table 3 The top 20 up-regulated and down-regulated IncRNAs in KGN/shPWRN2 cells

\begin{tabular}{|c|c|c|c|}
\hline \multicolumn{2}{|l|}{ Up-regulated IncRNAs } & \multicolumn{2}{|l|}{ Down-regulated IncRNAs } \\
\hline IncRNA ID (or known IncRNA name) & Fold-change & IncRNA ID (or known IncRNA name) & $\begin{array}{c}\text { Fold-change } \\
\text { nald }\end{array}$ \\
\hline XR_110904.1 & 5.369 & TCONS_00001136 & 4.033 \\
\hline XR_430233.1 & 4.498 & ENST00000557828.1 & 3.875 \\
\hline ENST00000584157.1 & 4.485 & RNA143578 & 3.422 \\
\hline RNA147334|p0438_imsncRNA843 & 4.185 & RNA143598 & 3.292 \\
\hline uc003qmi.3 & 4.118 & RNA146915|p0019_imsncRNA52 & 3.097 \\
\hline ENST00000543573.1 & 4.072 & TCONS_00019731 & 3.092 \\
\hline TCONS_00012676 & 3.934 & TCONS_00025502 & 3.088 \\
\hline ENST00000500823.2(GS1-24F4.2) & 3.730 & HIT000271588 & 3.068 \\
\hline ENST00000428520.2(LINC00710) & 3.440 & ENST00000567517.1(LINC00515) & 3.029 \\
\hline ENST00000502766.2 & 3.253 & RNA147684|p0788_imsncRNA576 & 2.963 \\
\hline ENST00000608299.1 & 3.252 & HIT000468529 & 2.883 \\
\hline ENST00000607195.1 & 3.047 & TCONS_00019573 & 2.882 \\
\hline XR_245201.2 & 3.035 & ENST00000452741.1 & 2.725 \\
\hline uc.311- & 3.034 & RNA143600 & 2.707 \\
\hline TCONS_00009112 & 3.013 & TCONS_00010743 & 2.638 \\
\hline ENST00000570809.1 & 2.997 & TCONS_00010435 & 2.628 \\
\hline uc.211- & 2.975 & ENST00000489626.1 & 2.551 \\
\hline ENST00000602984.1(MIR137HG) & 2.960 & uc022caj.1 & 2.548 \\
\hline TCONS_00000870 & 2.943 & ENST00000566245.1(PWNR2) & 2.526 \\
\hline ENST00000513586.1 & 2.928 & ENST00000439960.1 & 2.445 \\
\hline
\end{tabular}

Table 4 The top 20 up-regulated and down-regulated mRNAs in KGN/shPWRN2 cells

\begin{tabular}{|c|c|c|c|c|c|}
\hline \multicolumn{3}{|c|}{ Up-regulated mRNAs } & \multicolumn{3}{|c|}{ Down-regulated mRNAs } \\
\hline GeneSymbol & Genbank Accession & Fold-change & GeneSymbol & Genbank Accession & Fold-change \\
\hline CACTIN & NM_021231 & 5.976 & FTH1 & NM_002032 & 7.358 \\
\hline ND3 & HV963894 & 4.613 & FAM154A & NM_153707 & 3.460 \\
\hline NUB1 & NM_016118 & 4.417 & MOP-1 & AB014771 & 3.421 \\
\hline CCDC6 & NM_005436 & 3.410 & MARVELD2 & AK055094 & 3.176 \\
\hline MLF1 & NM_022443 & 3.227 & RPL19 & NM_000981 & 3.175 \\
\hline GPI & NM_000175 & 3.108 & CCNG2 & NM_004354 & 3.162 \\
\hline ADAM11 & NM_002390 & 3.066 & TMEM120B & NM_001080825 & 3.090 \\
\hline SYNGR4 & NM_012451 & 2.916 & SNX22 & XM_005254677 & 3.058 \\
\hline TMEM234 & NM_019118 & 2.855 & RGS4 & BC000737 & 3.051 \\
\hline PHIP & NM_017934 & 2.711 & FBRSL1 & NM_001142641 & 2.985 \\
\hline HIF3A & XR_243952 & 2.685 & MB21D1 & NM_138441 & 2.961 \\
\hline NIP7 & NM_016101 & 2.644 & ND4 & AK097322 & 2.779 \\
\hline C11orf45 & NM_145013 & 2.636 & FMNL1 & NM_005892 & 2.754 \\
\hline WWA1 & NM_022834 & 2.565 & $\mathrm{NHS}$ & NM_198270 & 2.753 \\
\hline ZSCAN32 & NM_017810 & 2.562 & SEMA6B & NM_032108 & 2.752 \\
\hline $\mathrm{RPPH} 1$ & CA413366 & 2.553 & RPL32 & NM_001007074 & 2.729 \\
\hline EPHA4 & NM_004438 & 2.551 & TLE6 & NM_024760 & 2.564 \\
\hline CCDC102B & NM_024781 & 2.403 & GRIP2 & NM_001080423 & 2.545 \\
\hline NOS1AP & NM_014697 & 2.378 & LOC100287036 & NM_001242885 & 2.454 \\
\hline CEP70 & NM_024491 & 2.377 & RPS2 & NM_002952 & 2.372 \\
\hline
\end{tabular}


qRT-PCR data of PWRN2 expression levels in the KGN/ shPWRN2 groups, indicating the reliability of lncRNA + mRNA microarray data.

\section{GO and KEGG pathway analyses}

The genes regulated by PWRN2 were selected to identify the associated signalling pathways and biological functions. Regarding GO annotation, the biological processes, cellular components and molecular function associated with the target genes are presented in Additional file 2: Table S2. The GO analysis showed that the most significantly altered biological processes were nuclear-transcribed mRNA catabolic process, RNA catabolic process, SRP-dependent co-translational protein targeting to the membrane, protein targeting to the ER and others. Moreover, the KEGG pathway analysis demonstrated that the most significantly altered pathways were mRNA surveillance pathways, carbon metabolism, nitrogen metabolism and axon guidance (Additional file 3: Table S3). These results indicated that PWRN2-regulated genes were mostly involved in different metabolic pathways, thereby confirming that PCOS is a complex metabolic disease.

\section{Construction of a PWRN2-regulated ceRNA network based on three microarray datasets}

Recent studies reported that lncRNAs have been reported to function as competing endogenous RNAs [33] by mopping up miRNAs, thereby regulating their function [34]. Given that lncRNAs can interact with miRNAs through their MREs within a ceRNA network [23], we searched for putative miRNAs with MREs in PWRN2 by using miRanda software. A total of 69 miRNAs were predicted to possess PWRN2 binding sites. The potential target mRNAs of these 69 miRNAs were also predicted (Additional file 4: Table S4).

We further compared the 69 potential PWRN2-regulated miRNAs with the differentially expressed miRNAs (21 up-regulated and 38 down-regulated) in the cumulus cells of patients with PCOS [25]. Two miRNAs (miR-92b-3p and miR-365b-5p) were found. PWRN2 was up-regulated in PCOS cumulus cells in our previous report [19], and the miRNAs (miR-92b-3p and miR-365b-5p) that possess PWRN2 binding sites were down-regulated [25]. Hence, PWRN2 may function as ceRNA to regulate miRNAs (miR-92b-3p and miR-365b-5p) in cumulus cells in PCOS.

By comparing the potential target genes of miR-92b-3p and miR-365b-5p with the PWRN2-regulated mRNAs identified by KGN/shPWRN2 microarray, we found 12 mRNAs, including UPF2, VSIG10, CCDC144A, LRRC1, ZNF654, FHL2, NOS1AP, TMEM120B, GPI, CCDC6, RCAN3 and AMOTL1. Thus, a credible PWRN2-miRNA-mRNA ceRNA network was constructed using the combined analyses of the three microarrays (lncRNA+mRNA microarray in PCOS, miRNA microarray in PCOS and lncRNA+mRNA microarray in KGN/shPWRN2; Figs. 3A, B).

According to our previous analysis of lncRNA and mRNA microarray data, PWRN2 and TMEM120B were over-expressed in PCOS cumulus cells [19]. In this study, the expression level of TMEM120B was down-regulated in KGN/shPWRN2 cells. The results indicated that the expression trends of PWRN2 and TMEM120B were consistent with PCOS. Moreover, miR-92b, which was predicted to process the MREs in PWRN2 and to have a binding site in the $3^{\prime}$-UTR of TMEM120B, was down-regulated in PCOS cumulus cells (Table 5). Therefore, we regarded the PWRN2-miR-92b-TMEM120B ceRNA network as more reasonable and credible.

\section{Co-expression characteristics of genes of the PWRN2-miR- 92b-3p-TMEM120B ceRNA network in PCOS}

The expression of the three candidate genes (PWRN2, miR-92b-3p and TMEM120B) in cumulus cell according to oocyte nuclear maturity $\left(\mathrm{CC}_{\mathrm{MI} / \mathrm{GV}}\right.$ and $\left.\mathrm{CC}_{\mathrm{MII}}\right)$ in patients with PCOS $(n=30)$ were detected by qRT-PCR to validate the potential ceRNA network. Based on comparison between $\mathrm{CC}_{\mathrm{MII}}$ and $\mathrm{CC}_{\mathrm{MI} / \mathrm{GV}}$ groups, the mean transcript level of miR-92b-3p in $C_{M I I}$ group was significantly 4.95 -fold higher than that in the $\mathrm{CC}_{\mathrm{MI} / \mathrm{GV}}$ group $(0.99 \pm 0.14$ versus $0.20 \pm 0.03)$ (Fig. $4 \mathrm{~A})$; by contrast, the expression levels of the other genes (PWRN2 and TMEM120B) significantly decreased in the $C_{\mathrm{MII}}$ samples, with 5.23- and 2.73-fold decrease observed for PWRN2 (0.98 \pm 0.05 versus $5.13 \pm 0.75)$ (Fig. 4B) and TMEM120B (1.0 \pm 0.06 versus $2.73 \pm 0.85)$ (Fig. 4C), respectively. A significant positive correlation was observed between PWRN2 and TMEM120B (Fig. 4D). The co-expression characteristics observed were in accordance with the ceRNA hypothesis.

\section{Confirmation of the direct interactions of the candidate genes of the PWRN2-miR-92b-3p-TMEM120B ceRNA network}

According to the prediction results, PWRN2 has one putative miR-92b-3p binding site. We recombined the PWRN2 cDNA (PWRN2-WT) and mutational cDNA (PWRN2-MUT) with the presumed miR-92b-3p deleted recognition sequences downstream the luciferase reporter gene (Fig. 5A). The vectors were transfected in PCOS cumulus cells along with the corresponding miRNA mimics. After miR-92b-3p mimics transfection, luciferase activity was reduced by $60 \%$ compared with the control miRNA (Fig. 5B). Hence, miR-92b-3p directly binds to PWRN2. 
A

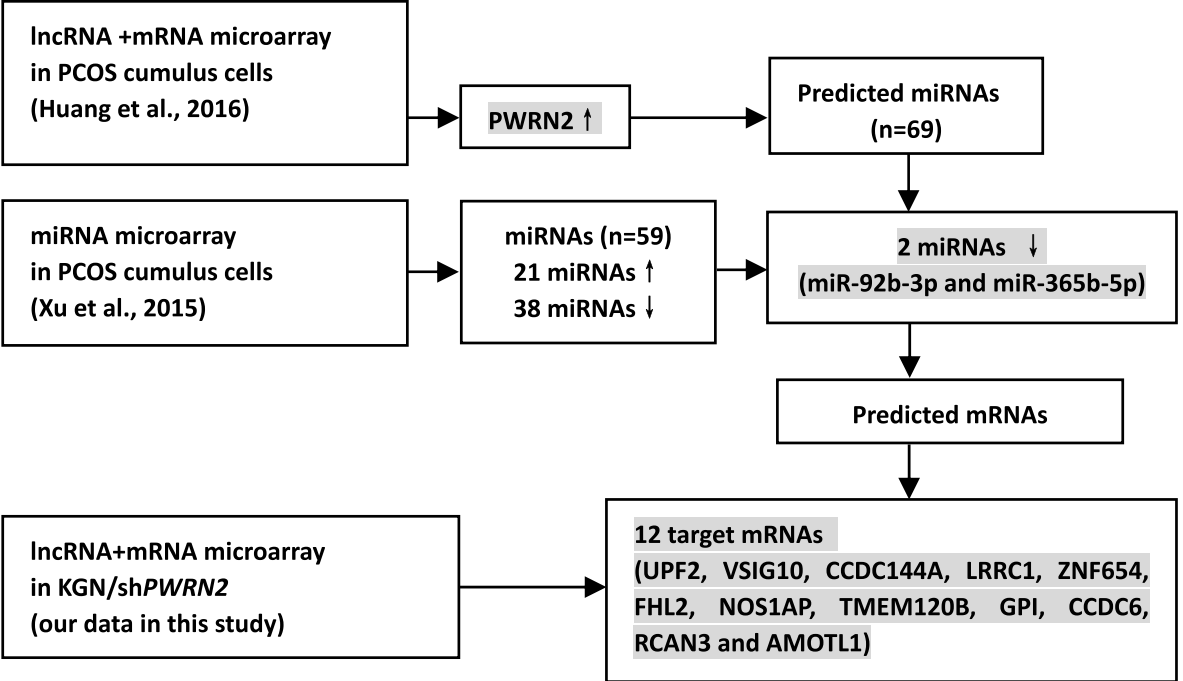

B

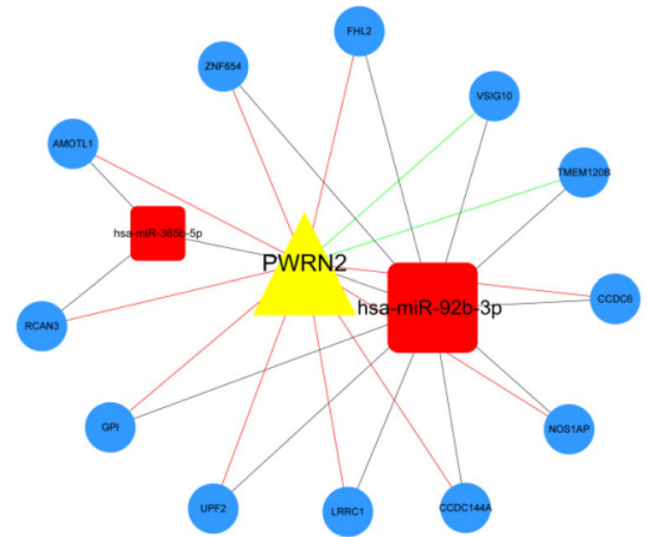

Fig. 3 Construction of a PWRN2-mediated ceRNA network based on microarray datasets. a Flow chart of building a PWRN2-mediated ceRNA network based on three microarray datasets: IncRNA+mRNA microarray in PCOS cumulus cells, miRNA microarray in PCOS cumulus cells and IncRNA+mRNA microarray in KGN/shPWRN2. b PWRN2-mediated ceRNA network. The ceRNA network is based on IncRNA/miRNA, IncRNA/mRNA and miRNA/mRNA interactions. In this network, black edges represent sequence matching, green edges represent positive correlations and red edges represent negative correlations

To determine whether TMEM120B is a direct target of miR-92b-3p, we constructed luciferase reporter constructs by cloning the DNA segment-encoding part of the WT or mutant (which cannot bind miR-92b-3p) 3' UTR of TMEM12OB mRNA downstream of the Renilla luciferase gene (Fig. 5A) and transfected them with miR-92b-3p mimics into the PCOS cumulus cells. As shown in Fig. $5 \mathrm{C}$, the relative luciferase activity significantly decreased in cumulus cells co-transfected with the TMEM120B WT constructs and miR-92b-3p mimics but not in cells co-transfected with the TMEM120B mutant constructs and miR-92b-3p mimics. Thus, miR-92b-3p suppressed the expression of TMEM120B in the cumulus cells by directly binding to the 3'UTR of TMEM120B mRNA.

\section{Discussion}

PWRN2 was up-regulated in PCOS cumulus cells and could be involved in oocyte development [19]. In the present study, we proved that PWRN2 is associated with oocyte nuclear maturation in patients with PCOS, in contrast to that in normal patients. Hence, PWRN2 plays important roles during the oocyte development in PCOS.

To elucidate the roles of PWRN2 in oocyte development, we identified PWRN2-regulated genes in KGN 
Table 5 The expression levels of PWRN2, miR-92b, and TMEM120B in three microarray data

\begin{tabular}{llll}
\hline Microarray data & PWRN2 & miR-92b & TMEM120B \\
\hline $\begin{array}{l}\text { IncRNA+mRNA array in PCOS cumulus cells } \\
\text { (Huang et al., 2016) }\end{array}$ & Up-regulated FC $=3.11$ & no & Up-regulated FC $=2.02$ \\
$\begin{array}{l}\text { miRNA array in PCOS cumulus cells } \\
\text { (Xu et al., 2015) }\end{array}$ & no & Down-regualted FC $=-2.27$ & no \\
$\begin{array}{l}\text { IncRNA+mRNA array in KGN/shPWRN2 array } \\
\text { (this study) }\end{array}$ & Down-regulated FC $=-2.52$ & no & Down-regulated FC $=-3.09$ \\
\hline
\end{tabular}

cells by using RNA interference technology. A total of 176 lncRNAs and 131 mRNAs were identified and determined to be regulated by PWRN2.The GO and KEGG pathway analyses of these DEGs showed that the differentially expressed genes were mostly involved in various catabolic processes (e.g. nuclear-transcribed mRNA and RNA catabolic processes) and metabolism pathways (e.g. carbon and nitrogen metabolism). These results indicated that PWRN2 may play an important role in metabolic processes. Metabolic abnormalities in cumulus cells in PCOS change the follicular microenvironment during oocyte development and subsequently affect oocyte quality $[35,36]$. This deduction is consistent with PCOS because it is a metabolic disease often accompanied by poor oocyte developmental potential.
Although potential target genes of PWRN2 were identified, the molecular mechanism of PWRN2 remains largely unknown. Several recent reports provided support for the ceRNA hypothesis, which accounts for the function of a substantial proportion of uncharacterised lncRNAs [37-40]. To examine if PWRN2 functions as a ceRNA, we developed a new method for elucidating specific lncRNA-mediated ceRNA networks. The most widely used methods for exploring potential lncRNA-miRNA-mRNA networks largely depend on shared MREs that are predicted by miRNA target discovery algorithms [41, 42]. We predicted miRNAs that possess PWRN2 binding sites and potential miRNA-mRNA pairs. We then obtained PWRN2-mediated miRNAs by combining the analyses of predicted
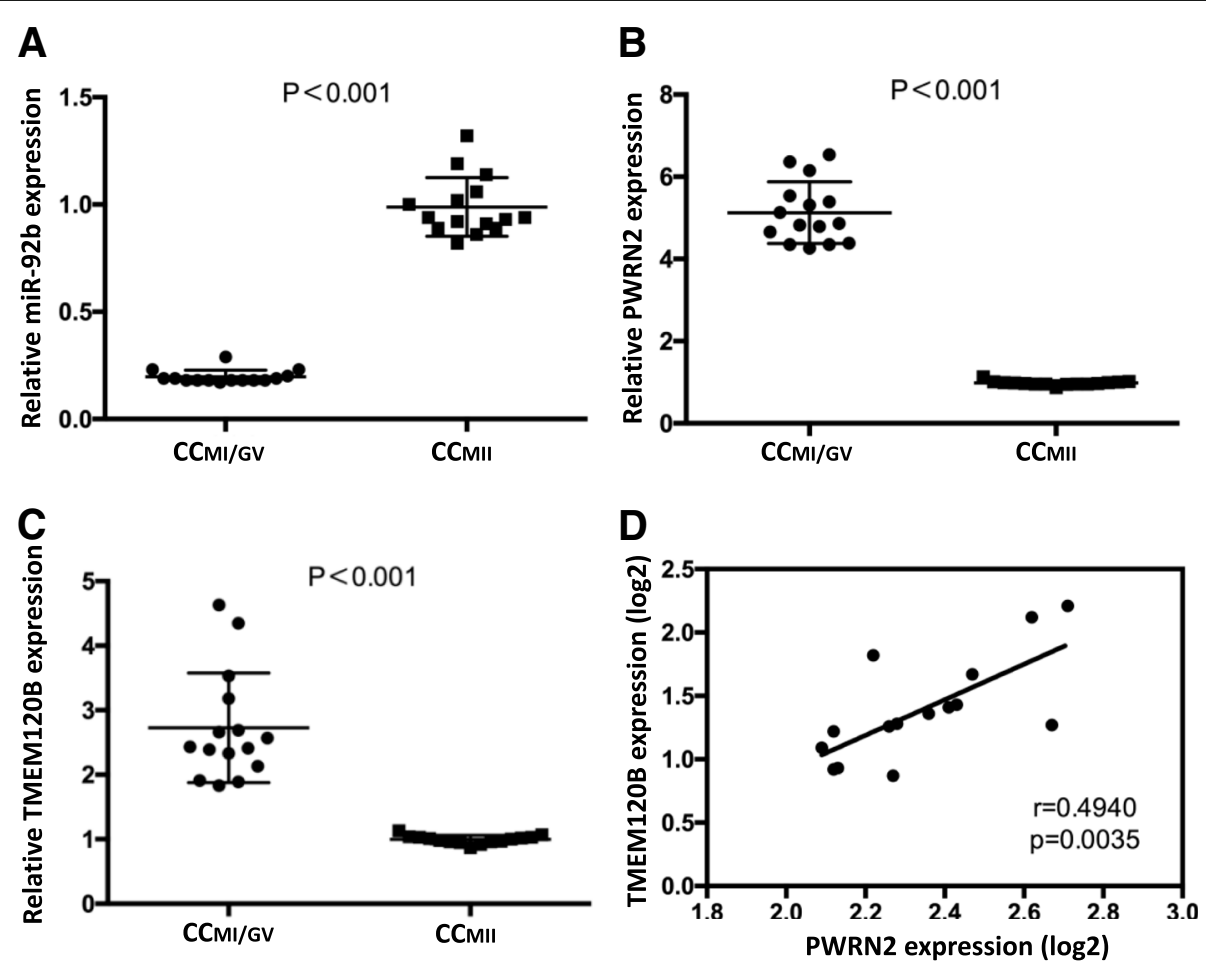

Fig. 4 Transcripts levels of the candidate genes of PWRN2-miR-92b-3p-TMEM120B ceRNA network according to oocyte nuclear maturity in patients with PCOS $(n=30)$. The transcript levels of miR-92b-3p (a), PWRN2 (b) and TMEM120B (c) were detected by qRT-PCR. $\mathbf{d}$ Relationship between the expression levels of PWRN2 and TMEM120B. The signal intensity for the genes is shown on the $y$-axis in arbitrary units determined by qRT-PCR analysis. GAPDH was used as internal control for PWRN2 and TMEM120B, while U6 was used as internal control for miR-92b-3p. * indicates a significant difference in gene expression between CC categories (** $P<0.01,{ }^{*} P<0.05$ ). The results were presented as means \pm SEM. $\mathrm{CC}_{\text {MI/GV: }}$ cumulus cells from oocyte at the MI or GV stage; $\mathrm{CC}_{\text {MII: }}$ cumulus cells from oocyte at the MII stage 


\section{A}

\begin{tabular}{|c|c|}
\hline $\begin{array}{l}\text { PWRN2 MUT: } \\
P W R N 2 \text { WT: }\end{array}$ & $\begin{array}{l}\text { 5'-atctgtGGCCCTGtgtgcagtctg-3' } \\
\text { 5'-atctgtCCGGGACtgtgcagtctg-3' }\end{array}$ \\
\hline hsa-miR-92b-3p: & 3'-ccuccGGCCCUGcuCACGUUAu-5' \\
\hline TMEM120B WT: & 5'- gatggggcagcctccctgGTGCAATc-3' \\
\hline TMEM120B MUT: & 5'- gatggggcagcctccctgCACGTTAc-3' \\
\hline
\end{tabular}
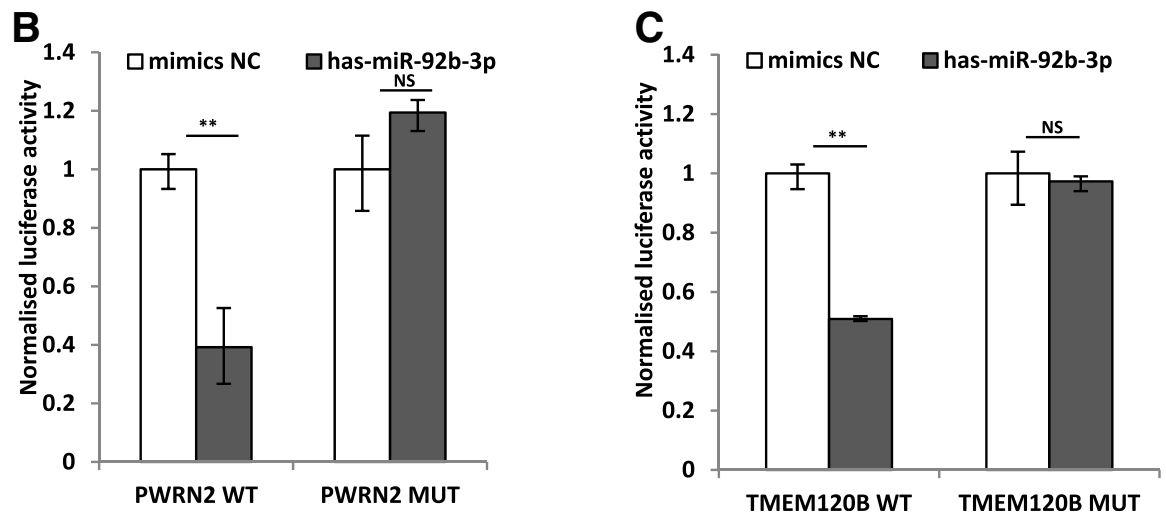

Fig. 5 Validation miR-92b-3p directly binds to PWRN2 and the direct interaction between miR-92b-3p and its predictive target gene (TMEM120B) by the dual luciferase activity assay. a Interaction regions of PWRN2/miR-92b-3p and miR-92b-3p/TMEM120B predicted by miRanda software. MiR$92 \mathrm{~b}-3 \mathrm{p}$, which is predicted to process the MREs in PWRN2, also has a binding site in the $3^{\prime}-$ UTR of TMEM120B. $\mathbf{b}$ The relative luciferase activity was assayed following co-transfection of miR-92b-3p mimics with the constructs encoding the wild-type or mutant PWRN2 cDNA into the cumulus cells. c The relative luciferase activity was assayed following co-transfection of miR-92b-3p mimics with the constructs encoding the wild-type or mutant miR-92b-3p binding site of TMEM120B 3'-UTR into the cumulus cells. The constructed reporter vectors (WT or MUT) are shown on the xaxis, and the $y$-axis represents the normalised luciferase activity (firefly luciferase activities were normalised to renilla luciferase activities). The experiments were performed independently in triplicate. ${ }^{*}$ indicates $P<0.05,{ }^{* *}$ indicates $P<0.01$, NS indicates no significant difference. The results are presented as means \pm S.E.M

miRNAs and miRNA microarray data from previous report [25]. Meanwhile, PWRN2-regulated mRNAs were obtained by comparing potential miRNA-target mRNA with the microarray data from KGN/shPWRN2 in the present study. Finally, we incorporated a bioinformatic prediction tool to construct a PWRN2-miRNA-mRNA ceRNA network. Based on the dual-luciferase activity assay, the ceRNA network is reliable because it is based on datasets from three microarrays.

Furthermore, we investigated PWRN2 and miR-92b$T M E M 120 B$ pair and validated their expression levels in cumulus cells according to oocyte nuclear maturity $\left(\mathrm{CC}_{\mathrm{MI} / \mathrm{GV}}\right.$ and $\left.\mathrm{CC}_{\mathrm{MII}}\right)$ of patients with PCOS. The results were in accordance with the ceRNA hypothesis. In this regard, PWRN2 functions as ceRNA to reduce the availability of miR-92b-3p for TMEM120B target binding. $T M E M 120 B$ is a fat-specific nuclear envelope transmembrane protein that may play a contributory role in adipogenesis [43]. In PCOS, up-regulated TMEM120B will promote adipocyte differentiation/metabolism and induce obesity. Severe obesity has been shown to be associated with a high prevalence of spindle anomalies and nonaligned chromosomes in failed fertilised oocytes [44]. Thus, the ceRNA network of PWRN2-miR-92b-T$M E M 120 B$ provides a basis for explaining the poor quality of oocyte in patients with PCOS.

Our study presents limitations. Firstly, we cannot identify large lncRNA-miRNA-mRNA ceRNA networks by combining microarray datasets. Moreover, the molecular roles of the PWRN2-miR-92b-TMEM12OB network in oocyte development in PCOS require further investigation. Secondly, a large number of samples must be analysed to validate the ceRNA network in PCOS. Our future work will further validate the PWRN2-miR-92b-TMEM120B network and elucidate its role in the pathogenesis of PCOS, especially in oocyte development.

In conclusion, our results proved that lncRNA (PWRN2) is associated with oocyte nuclear maturation in PCOS. The constructed PWRN2-miR-92b-TMEM120B ceRNA network based on three microarray datasets indicated that 
PWRN2 functions as ceRNA to reduce the availability of miR-92b-3p for TMEM120B target binding during oocyte nuclear maturation in PCOS. This ceRNA network provides new information and helps clarify the metabolic disorder that induces abnormal oocyte development in PCOS.

\section{Additional files}

Additional file 1: The primers used in this study. (XLSX $12 \mathrm{~kb}$ )

Additional file 2: $\mathrm{GO}$ annotation of the target genes regulated by PWRN2. (XLSX $283 \mathrm{~kb}$ )

Additional file 3: KEGG pathway analysis of the target genes regulated by PWRN2. (XLSX $35 \mathrm{~kb}$ )

Additional file 4: The potential target mRNAs of putative miRNAs with MREs in PWRN2. (XLSX $97 \mathrm{~kb}$ )

\section{Abbreviations}

CCs: Cumulus cells; ceRNA: competing endogenous RNAs; COC: Cumulusoocyte complex; IncRNA: long non-coding RNAs; PCOS: Polycystic ovary syndrome; PWRN2: Prader-Willi region non-protein coding RNA 2

\section{Acknowledgements}

The authors gratefully acknowledge the CapitalBio Corporation for conducting the RNA extractions and microarrays.

\section{Funding}

This study was supported by the National Natural Science Foundation of China (Grant 81401172 and 81771532), Special Fund for Clinical Medicine Scientific Research of Chinese Medical Association (Grant 16020180634). Shanghai municipal medical and health discipline construction projects (Grant 2017ZZ02015).

\section{Availability of data and materials}

All data generated or analysed during this study are included in this published article [and its supplementary information files].

\section{Authors' contributions}

Xin Huang conducted the experiments and generated the manuscript. Xiaoming Teng contributed to study design and data interpretation. Jiaping Pan contributed to data interpretation and manuscript editing. Bi Wu contributed to study design, data interpretation and manuscript preparation and editing. All authors read and approved the final manuscript.

\section{Ethics approval and consent to participate}

The study was approved by the Institutional Ethical Review Board of Tongji University School of Medicine on the 18th March 2015 (Reference: TJUSM2015-0318).

Written informed consent was obtained from all patients and the study was approved by the Ethics Review Board of Shanghai First Maternity and Infant Hospital on 20th November 2015 (Reference: 2015-RM-1120).

\section{Consent for publication}

Not applicable.

\section{Competing interests}

The authors declare no conflicts of interest.

\section{Publisher's Note}

Springer Nature remains neutral with regard to jurisdictional claims in published maps and institutional affiliations.
Received: 29 March 2018 Accepted: 25 July 2018

Published online: 03 August 2018

\section{References}

1. Shi Y, Zhao H, Cao Y, Yang D, Li Z, Zhang B, Liang X, Li T, Chen J, Shen J, et al. Genome-wide association study identifies eight new risk loci for polycystic ovary syndrome. Nat Genet. 2012:44:1020-5.

2. Diao FY, Xu M, Hu Y, Li J, Xu Z, Lin M, Wang L, Zhou Y, Zhou Z, Liu J, Sha J. The molecular characteristics of polycystic ovary syndrome (PCOS) ovary defined by human ovary cDNA microarray. J Mol Endocrinol. 2004;33:59-72.

3. Revised 2003 Consensus on diagnostic criteria and long-term health risks related to polycystic ovary syndrome. Fertil Steril. 2004;(81):19-25.

4. Kandaraki E, Christakou C, Diamanti-Kandarakis E. Metabolic syndrome and polycystic ovary syndrome... and vice versa. Arq Bras Endocrinol Metabol. 2009;53:227-37.

5. Carmina E. Cardiovascular risk and events in polycystic ovary syndrome. Climacteric. 2009;12(Suppl 1):22-5.

6. Wild S, Pierpoint T, Jacobs H, McKeigue P. Long-term consequences of polycystic ovary syndrome: results of a 31 year follow-up study. Hum Fertil (Camb). 2000:3:101-5.

7. Diamanti-Kandarakis E, Piperi C. Genetics of polycystic ovary syndrome: searching for the way out of the labyrinth. Hum Reprod Update. 2005;11: 631-43.

8. Vink JM, Sadrzadeh S, Lambalk CB, Boomsma DI. Heritability of polycystic ovary syndrome in a Dutch twin-family study. J Clin Endocrinol Metab. 2006;91:2100-4

9. Wood JR, Ho CK, Nelson-Degrave VL, McAllister JM, Strauss JF 3rd. The molecular signature of polycystic ovary syndrome (PCOS) theca cells defined by gene expression profiling. J Reprod Immunol. 2004;63:51-60.

10. Jansen E, Laven JS, Dommerholt HB, Polman J, van Rijt $C_{\text {, van }}$ den Hurk $C$, Westland J, Mosselman S, Fauser BC. Abnormal gene expression profiles in human ovaries from polycystic ovary syndrome patients. Mol Endocrinol. 2004:18:3050-63.

11. Oksjoki S, Soderstrom M, Inki P, Vuorio E, Anttila L. Molecular profiling of polycystic ovaries for markers of cell invasion and matrix turnover. Fertil Steril. 2005;83:937-44.

12. Wood JR, Dumesic DA, Abbott DH, Strauss JF 3rd. Molecular abnormalities in oocytes from women with polycystic ovary syndrome revealed by microarray analysis. J Clin Endocrinol Metab. 2007:92:705-13.

13. Kenigsberg S, Bentov Y, Chalifa-Caspi V, Potashnik G, Ofir R, Birk OS. Gene expression microarray profiles of cumulus cells in lean and overweightobese polycystic ovary syndrome patients. Mol Hum Reprod. 2009;15:89-103.

14. Mohamed-Hussein ZA, Harun S. Construction of a polycystic ovarian syndrome (PCOS) pathway based on the interactions of PCOS-related proteins retrieved from bibliomic data. Theor Biol Med Model. 2009;6:18.

15. Mattick JS. Non-coding RNAs: the architects of eukaryotic complexity. EMBO Rep. 2001;2:986-91.

16. Ponting CP, Belgard TG. Transcribed dark matter: meaning or myth? Hum Mol Genet. 2010;19:R162-8.

17. Ponting $\mathrm{CP}$, Oliver PL, Reik W. Evolution and functions of long noncoding RNAs. Cell. 2009;136:629-41.

18. Moran I, Akerman I, van de Bunt M, Xie R, Benazra M, Nammo T, Arnes L, Nakic N, Garcia-Hurtado J, Rodriguez-Segui S, et al. Human beta cell transcriptome analysis uncovers IncRNAs that are tissue-specific, dynamically regulated, and abnormally expressed in type 2 diabetes. Cell Metab. 2012; 16:435-48.

19. Huang X, Hao C, Bao H, Wang M, Dai H. Aberrant expression of long noncoding RNAs in cumulus cells isolated from PCOS patients. J Assist Reprod Genet. 2016;33:111-21.

20. Wawrzik M, Spiess AN, Herrmann R, Buiting K, Horsthemke B. Expression of SNURF-SNRPN upstream transcripts and epigenetic regulatory genes during human spermatogenesis. Eur J Hum Genet. 2009;17:1463-70.

21. Christakou C, Diamanti-Kandarakis E. Polycystic ovary syndrome-phenotypes and diagnosis. Scand J Clin Lab Invest Suppl. 2014;244:18-22. discussion 21

22. Fux Otta C, Fiol de Cuneo M, Szafryk de Mereshian P. polycystic ovary syndrome: physiopathology review. Rev Fac Cien Med Univ Nac Cordoba. 2013;70:27-30

23. Salmena L, Poliseno L, Tay Y, Kats L, Pandolfi PP. A ceRNA hypothesis: the Rosetta stone of a hidden RNA language? Cell. 2011;146:353-8. 
24. Tay Y, Rinn J, Pandolfi PP. The multilayered complexity of ceRNA crosstalk and competition. Nature. 2014;505:344-52.

25. Xu B, Zhang YW, Tong XH, Liu YS. Characterization of microRNA profile in human cumulus granulosa cells: identification of microRNAs that regulate notch signaling and are associated with PCOS. Mol Cell Endocrinol. 2015; 404:26-36.

26. Huang X, Liu C, Hao C, Tang Q, Liu R, Lin S, Zhang L, Yan W. Identification of altered microRNAs and mRNAs in the cumulus cells of PCOS patients: miRNA-509-3p promotes oestradiol secretion by targeting MAP3K8. Reproduction. 2016;151:643-55.

27. Huang $X$, Hao C, Shen $X$, Liu X, Shan Y, Zhang Y, Chen L. Differences in the transcriptional profiles of human cumulus cells isolated from MI and MII oocytes of patients with polycystic ovary syndrome. Reproduction. 2013;145: 597-608.

28. Huang X, Hao C, Shen X, Zhang Y, Liu X. RUNX2, GPX3 and PTX3 gene expression profiling in cumulus cells are reflective oocyte/embryo competence and potentially reliable predictors of embryo developmental competence in PCOS patients. Reprod Biol Endocrinol. 2013;11:109.

29. Livak KJ, Schmittgen TD. Analysis of relative gene expression data using real-time quantitative PCR and the 2(-Delta Delta C(T)) method. Methods. 2001;25:402-8.

30. Shen T, Liu K, Miao D, Cao R, Zhou B, Chen P. Lentivirus-mediated RNA interference against Japanese encephalitis virus infection in vitro and in vivo. Antivir Res. 2014:108:56-64.

31. Su Z, Zhi X, Zhang Q, Yang L, Xu H, Xu Z. LncRNA H19 functions as a competing endogenous RNA to regulate AQP3 expression by sponging miR-874 in the intestinal barrier. FEBS Lett. 2016;590:1354-64.

32. Huang S, Lu W, Ge D, Meng N, Li Y, Su L, Zhang S, Zhang Y, Zhao B, Miao J. A new microRNA signal pathway regulated by long noncoding RNA TGFB2OT1 in autophagy and inflammation of vascular endothelial cells. Autophagy. 2015;11:2172-83.

33. John-Aryankalayil M, Palayoor ST, Makinde AY, Cerna D, Simone CB 2nd, Falduto MT, Magnuson SR, Coleman CN. Fractionated radiation alters oncomir and tumor suppressor miRNAs in human prostate cancer cells. Radiat Res. 2012;178:105-17.

34. Shi X, Sun M, Liu H, Yao Y, Song Y. Long non-coding RNAs: a new frontier in the study of human diseases. Cancer Lett. 2013;339:159-66.

35. Arya BK, Haq AU, Chaudhury K. Oocyte quality reflected by follicular fluid analysis in poly cystic ovary syndrome (PCOS): a hypothesis based on intermediates of energy metabolism. Med Hypotheses. 2012;78:475-8.

36. Gluszak O, Stopinska-Gluszak U, Glinicki P, Kapuscinska R, Snochowska H, Zgliczynski W, Debski R. Phenotype and metabolic disorders in polycystic ovary syndrome. ISRN Endocrinol. 2012;2012:569862.

37. Thomson DW, Dinger ME. Endogenous microRNA sponges: evidence and controversy. Nat Rev Genet. 2016;17:272-83.

38. Tay Y, Karreth FA, Pandolfi PP. Aberrant ceRNA activity drives lung cancer. Cell Res. 2014;24:259-60.

39. Chen L, Wang W, Cao L, Li Z, Wang X. Long non-coding RNA CCAT1 acts as a competing endogenous RNA to regulate cell growth and differentiation in acute myeloid leukemia. Mol Cells. 2016;39:330-6.

40. Sun C, Li S, Zhang F, Xi Y, Wang L, Bi Y, Li D. Long non-coding RNA NEAT1 promotes non-small cell lung cancer progression through regulation of miR-377-3p-E2F3 pathway. Oncotarget. 2016;7:51784-814.

41. Song C, Zhang J, Liu Y, Pan H, Qi HP, Cao YG, Zhao JM, Li S, Guo J, Sun HL, Li CQ. Construction and analysis of cardiac hypertrophy-associated IncRNAmRNA network based on competitive endogenous RNA reveal functional IncRNAs in cardiac hypertrophy. Oncotarget. 2016;7:10827-40.

42. Liu XH, Sun M, Nie FQ, Ge YB, Zhang EB, Yin DD, Kong R, Xia R, Lu KH, Li JH, et al. Lnc RNA HOTAIR functions as a competing endogenous RNA to regulate HER2 expression by sponging miR-331-3p in gastric cancer. Mol Cancer. 2014:13:92.

43. Batrakou DG, de Las Heras Jl, Czapiewski R, Mouras R, Schirmer EC. TMEM120A and B: nuclear envelope transmembrane proteins important for adipocyte differentiation. PLoS One. 2015;10:e0127712.

44. Machtinger R, Combelles CM, Missmer SA, Correia KF, Fox JH, Racowsky C. The association between severe obesity and characteristics of failed fertilized oocytes. Hum Reprod. 2012;27:3198-207.

\section{Ready to submit your research? Choose BMC and benefit from:}

- fast, convenient online submission

- thorough peer review by experienced researchers in your field

- rapid publication on acceptance

- support for research data, including large and complex data types

- gold Open Access which fosters wider collaboration and increased citations

- maximum visibility for your research: over $100 \mathrm{M}$ website views per year

At BMC, research is always in progress.

Learn more biomedcentral.com/submissions 